\title{
Uma ontologia de referência para arquiteturas pedagógicas
}

\author{
Pedro David Netto Silveira ${ }^{1}$, Rosane Aragon ${ }^{3}$ \\ Davidson Cury ${ }^{2}$, Crediné Silva de Menezes ${ }^{3}$,
}

\author{
${ }^{1}$ Núcleo de Informática \\ Instituto Federal do Espírito Santo (UFES) - Alegre, ES - Brasil
}

${ }^{2}$ Departamento de Informática

Universidade Federal do Espírito Santo (UFES) - Vitória, ES - Brasil

${ }^{3}$ Departamento de Informática na Educação

Universidade Federal do Rio Grande do Sul (UFRGS) - Porto Alegre, RS - Brasil

\{pedro.dns, dedecury, credine, rosane.aragon\}@gmail.com

\begin{abstract}
The digital culture advent provides us with an opportune moment to encourage interactions that enhance situations to favor the knowledge construction. However, the development of innovative pedagogical proposals requires theoretical support that guarantees the effectiveness of new initiatives. In this context, Pedagogical Architectures have proven to be adequate for the development of solutions based on open and flexible approaches. Despite the numerous existing works supported by this theoretical conception, this domain still lacks a shared and formal conceptualization. This work presents an ontology for pedagogical architectures, its validation and finally we show how to use it in formulating new architectures.
\end{abstract}

Resumo. $O$ advento da cultura digital nos proporciona um momento oportuno para favorecer interações que potencializem situações propícias à construção de conhecimento. Entretanto, a elaboração de propostas pedagógicas inovadoras requer um suporte teórico que garanta a efetividade de novas iniciativas. Neste contexto, as Arquiteturas Pedagógicas têm se mostrado adequadas para a elaboração de soluções baseadas em abordagens abertas e flexíveis. Apesar dos inúmeros trabalhos existentes apoiados nesta concepção teórica, este domínio ainda carece de uma conceitualização compartilhada e formal. Este trabalho apresenta uma ontologia para arquiteturas pedagógicas, sua validação e por fim mostramos como usá-la na formulação de novas arquiteturas.

\section{Introdução}

As possibilidades abertas pelo advento da cultura digital, principalmente com a ubiquidade criada pelas tecnologias, nos proporciona uma facilidade considerável para flexibilizar nossas interações e ajudar no registro e gerenciamento delas. Isto é muito importante para a educação.

Nas abordagens correntes para a inovação dos processos educacionais, potencializados pelas tecnologias digitais, destacam-se as metodologias ativas [Valente et al. 2017], que defendem o protagonismo dos estudantes nos processos de aprendizagem. No entanto, apenas o alto desenvolvimento tecnológico e a cultura digital em si, não são suficientes para promover uma educação de qualidade. Faz-se necessário um suporte teórico que garanta a efetividade de novas soluções. 
Buscando contribuir com a concepção de novas propostas pedagógicas alinhadas com os propósitos delineados pelas metodologias ativas, vemos surgir o conceito de Arquiteturas Pedagógicas, que oferecem um suporte teórico, considerando a epistemologia genética de Jean Piaget, a Pedagogia da Autonomia de Paulo Freire, e as ecologias da cognição de Gregory Bateson e da aprendizagem de Norman Jackson.

Existem muitos trabalhos à respeito do tema arquiteturas pedagógicas [Marcon et al. 2013]; [Aragón 2016]; [Carvalho et al. 2005]; [Menezes et al. 2021]; [Castro and Menezes 2011], inclusive com a criação de várias delas e seu devido suporte computacional. No entanto, esse domínio ainda carece de uma conceituação formal e compartilhada.

Para viabilizar a elaboração do suporte computacional de novas arquiteturas pedagógicas, este artigo propõe uma Ontologia de referência para Arquiteturas Pedagógicas (OAP), desenvolvida com a metodologia SABiO [Falbo 2014], e que apresenta os seguintes usos pretendidos: (i) apoiar a especialização e instanciação de novas Arquiteturas Pedagógicas (APs) e suas possíveis concepções tecnológicas e (ii) Fornecer um aparato conceitual para que não-especialistas do domínio de arquiteturas pedagógicas possam identificar se a estrutura processual que tem em mãos é realmente uma instância de arquitetura pedagógica.

O restante do documento está organizado da seguinte forma: Seção 2 contém o referencial teórico, a Seção 3 apresenta OAP incluindo a metodologia, o escopo e a sua formalização. Na Seção 4 apresentamos as tarefas de Validação e Verificação (V\&V) que realizamos em OAP e finalmente na Seção 5 apresentamos as considerações finais.

\section{Referencial Teórico}

Nesta seção abordaremos os conceitos necessários para o entendimento desta proposta. Destacamos principalmente (i) arquiteturas pedagógicas, sua conceituação e como a mediação pedagógica atua neste contexto; e (ii) ontologias, explicando qual sua função e como representá-las.

\subsection{Arquiteturas Pedagógicas}

A inserção das tecnologias digitais na educação encontra obstáculos na concepção da sua integração como uma simples soma de novos recursos tecnológicos às práticas pedagógicas transmissivas, ainda arraigadas no nosso cenário educacional. Buscando superar esse paradigma de base instrucionista, que se limita a produzir mais do mesmo, sem uma revisão nas ideias epistemológicas e pedagógicas que o sustenta, propomos uma abordagem de integração das tecnologias aos processos de aprendizagem baseada nos pressupostos da abordagem das ecologias cognitivas [Bateson 2000];[Lévy 1993] e da ecologia da aprendizagem [Jackson 2013].

Considerando a perspectiva ecológica na qual se inserem, as arquiteturas podem ser compreendidas como "microecossistemas cognitivos" [Aragón 2016], definidos pelas interrelações e dinâmicas que se estabelecem entre os diversos componentes. Conforme [Carvalho et al. 2005] e [Aragón 2016], as arquiteturas são definidas como "suportes estruturantes" para a aprendizagem que englobam diferentes componentes em interação: pedagogias relacionais, métodos ativos, abordagens cooperativas, tecnologias digitais, inteligência artificial e reconfigurações dos tempos e espaços de aprendizagem, entre outros. 
Além da ideia de interdependência, a concepção da ideia de arquiteturas encontra, ainda, suporte na articulação entre a concepção construtivista de aprendizagem [Piaget 2016] e a pedagogia da pergunta [Freire 2014]. Para aprender, os sujeitos precisam agir sobre os objetos (físicos, sociais etc.), mas para promover as interações é preciso que eles encontrem apoios para a sua ação. Não se trata de dar caminhos pré-definidos, mas sim oferecer um arcabouço (ou suporte) estruturante para que os caminhos se construam na dinâmica das interações (experimentações, debates em rede etc.).

As arquiteturas desafiam alunos e professores a desenvolverem um olhar interrogativo, curioso, e propõem deslocamentos importantes que envolvem (i) transformações nos papéis desempenhados tradicionalmente por professores e alunos e (ii) a revisão do sentido do uso das tecnologias nos processos educacionais.

Na proposta das arquiteturas o professor é convidado a transformar a sua perspectiva de transmissor de conteúdo para a de mediador das aprendizagens. Nesse contexto, as estratégias mediadoras seguem duas vertentes complementares: por um lado, a mediação busca desestabilizar os conhecimentos atuais promovendo os "conflitos cognitivos" e, por outro lado, oferecer apoios para as reconstruções, como por ex. orientações de estudos, disponibilização de materiais, incentivo à participação protagonista e à meta-reflexão. Cabe a função de mediação provocar o diálogo [Freire 2014] e a pesquisa a partir do campo de conhecimento atual, para que esse possa ser questionado e reconstruído.

Como as arquiteturas propõe a aprendizagem em rede e a horizontalidade das relações, a função de mediação encontra uma ressignificação a partir de uma política de participação que envolve a redução da centralidade do professor, abrindo espaço para que a mediação também ocorra entre os pares, ou seja, para que se estabeleça o que denominamos de "mediação distribuída" [Aragón 2016]. Essa forma de mediação distribuída oportuniza que os alunos exerçam protagonismos e responsabilidades para com os seus pares. Aprender a realizar essa forma de mediação se dá no "fazer", nas experiências que devem ser oportunizadas pela arquitetura, caracterizando o que tem sido nomeado mais amplamente como "aprender fazendo".

Conforme [Menezes et al. 2021], no contexto das arquiteturas, o suporte computacional é imprescindível. Não se trata de um uso periférico e dissociado de TI, mas sim de considerá-las na composição e dinâmica de um arcabouço estruturante para as aprendizagens, com destaque para a aprendizagem cooperativa. Ao mesmo tempo em que o suporte computacional oportuniza uma expansão das aprendizagens, garante que os tempos e espaços das interações sejam delimitados, conforme momentos/etapas definidas pelas estratégias pedagógicas que propõem alternâncias entre a ação individual e a coletiva.

Dessa forma, as tecnologias precisarão oferecer condições facilitadoras, tanto para o desenvolvimento das ações e mediações, quanto para a análise dos processos cognitivos. Para tal, é necessário que elas ofereçam suporte para a configuração do ambiente de aprendizagem, de tal forma que as atividades repetitivas possam ser repassadas para os componentes tecnológicos. Conforme a necessidade, o suporte computacional deverá possibilitar o uso de técnicas de inteligência artificial como apoio às mediações [Menezes et al. 2021]. 


\subsection{Ontologias}

Ontologia em informática está relacionada ao termo que é utilizado em filosofia: "uma teoria sobre os tipos de entidades e, especificamente, os tipos de entidades abstratas que devem ser admitidas em um sistema de linguagem" [Guizzardi 2007]. As ontologias nem sempre podem ser ideais. Portanto, uma definição geral (não ideal) para uma ontologia deve ser dada: "Uma ontologia é uma especificação conceitual que descreve o conhecimento sobre um domínio de forma que seja independente dos estados epistêmicos das coisas. Além disso, esta definição pretende restringir as possíveis interpretações do vocabulário de uma língua, de forma que seus modelos lógicos se aproximem o máximo possível do conjunto de estruturas universais pretendidas de uma conceituação desse domínio" [Guizzardi 2007].

As ontologias podem ser usadas para uma variedade de propósitos, incluindo o suporte à especificação de domínios ou de sistemas de computação complexos. Em geral, buscamos atingir os seguintes objetivos com uma ontologia: (i) ajudar as pessoas a compreender melhor uma determinada área do conhecimento; (ii) ajudar as pessoas a chegarem a um consenso na compreensão de uma área do conhecimento e (iii) ajudar as pessoas a compreender uma determinada área do conhecimento sem a necessidade de consultar um especialista nessa área [Duarte and Falbo 2000]

\section{Ontologia de referência para Arquiteturas Pedagógicas (OAP)}

Nesta seção apresentaremos uma proposta de ontologia de referência para o domínio de arquiteturas pedagógicas, explicando a metodologia utilizada, o levantamento de escopo e questões de competência e a formalização dela utilizando OntoUML.

\subsection{Metodologia}

O desenvolvimento da ontologia foi baseado no método SABiO (Systematic Approach for Building Ontologies) [Falbo 2014]. Escolhemos SABiO, principalmente por dois motivos: (i) por se tratar de um método sólido de engenharia de ontologias que fornece um conjunto de atividades e diretrizes para a especificação de ontologias, e (ii) por ser uma abordagem focada na criação de ontologias de domínio, que é o caso de OAP.

SABiO estabelece um processo composto por cinco fases: (i) Identificação da finalidade e dos usos pretendidos, bem como a definição de requisitos por meio do levantamento de questões de competência; (ii) Captura e formalização da ontologia; (iii) Projeto de refinamento de ontologias; (iv) Implementação usando uma linguagem operacional, por exemplo OWL, caso haja uso pretendido que exija uma ontologia operacional; e (v) Testes de verificação e validação de ontologias [Falbo 2014].

Para a formalização de OAP, utilizamos uma técnica baseada em UML 2.0, denominada OntoUML [Guizzardi 2007], que usa meta-propriedades ontológicas e a categorização de objetos propostos em UFO (Unified Foundational Ontology) [Guizzardi 2007]. Isso é feito com a introdução de estereótipos no diagrama de classes para obter uma caracterização ontológica. A Tabela 1 mostra os estereótipos usados por OAP.

Uma das grandes vantagens de usar OntoUML para uma representação visual de conceitos é a capacidade intrínseca da ferramenta de adicionar semântica ao cenário. Caso 
Tabela 1. Estereótipos OntoUML.

\begin{tabular}{|c|c|}
\hline Estereotipo & Descrição \\
\hline$<<$ kind $>>$ & Tipos rígidos* que provê critério de identidade para suas instâncias. \\
\hline$<<$ subkind $>>$ & Tipos rígidos* cujas instâncias especializam instâncias de $<<$ kind $>>$. \\
\hline$<<$ category $>>$ & $\begin{array}{l}\text { Tipos rígidos* que capturam propriedades comuns ou compartilhadas entre } \\
\text { entidades que possuem princípios de identidade diferentes. }\end{array}$ \\
\hline$<<$ role $>>$ & Tipos anti-rígidos instanciados dentro do escopo de uma relação. \\
\hline$<<$ phase $>>$ & $\begin{array}{l}\text { Tipos anti-rígidos que são instanciados por alterações nas propriedades } \\
\text { intrínsecas de tipos rígidos. }\end{array}$ \\
\hline$<<$ roleMixin $>>$ & Anti-rígidos que generalizam tipos com diferentes critérios de identidade. \\
\hline$<<$ relator $>>$ & $\begin{array}{l}\text { Tipo que reúne um relacionamento e cujas instâncias são existencialmente } \\
\text { dependentes de entidades relacionadas. }\end{array}$ \\
\hline$<<$ quality $>>$ & $\begin{array}{l}\text { Referem-se às características (ou atributos) associados a uma estrutura de } \\
\text { qualidade, ou seja, um conhecido espaço de valores, como cores. }\end{array}$ \\
\hline$<<$ mode $>>$ & $\begin{array}{l}\text { Referem-se a características (ou atributos) associados a algo que não pode } \\
\text { ser medido com valores, como intenção ou desejo. }\end{array}$ \\
\hline
\end{tabular}

a explicação fornecida nesta seção não seja suficiente para a compreensão do modelo conceitual de nossa proposta, recomendamos a leitura da página [ONTOUML 2018], que apresenta um manual completo para um estudo mais aprofundado sobre o assunto.

\subsection{Escopo e questões de competência}

O escopo da ontologia cobre os conceitos centrais sobre dois módulos: (i) arquiteturas pedagógicas e (ii) ambientes educacionais. Porém, por ser o segundo, um domínio muito amplo, não trazemos todos os aspectos, nos restringindo aos conceitos necessários para a explicação do domínio de arquiteturas pedagógicas.

No que diz respeito à criação de uma $\mathrm{AP}$, se faz necessário uma estrutura composta por: (i) Objetivo pedagógico que aponta qual a aprendizagem possibilitada pela AP; (ii) Atividades pedagógicas especialmente planejadas para atingir esse objetivo pedagógico; (iii) Método pedagógico a seguir; (iv) Plano de trabalho que estabelece a forma como cada atividade pedagógica será executada; e (v) recursos digitais cuidadosamente configurados para possibilitar o desenvolvimento das atividades planejadas [Castro and Menezes 2011]

Definido o escopo de OAP, identificamos as Questões de Competência (QC). Essas questões visam refinar o escopo da ontologia e orientar seu desenvolvimento. Elas também são usados como base para avaliar a qualidade da ontologia de referência. Apesar do escopo de OAP considerar conceitos de ambientes educacionais, vamos restringir a análise de competência ao módulo de AP. Assim, as seguintes QCs foram identificadas:

QC01. Quem participa de uma AP?

QC02. Quais são os elementos de uma AP?

QC03. Qual é o objetivo pedagógico de uma AP?

QC04. O que define como uma AP deve ser executada? 


\section{QC05. Uma AP pode ser apoiada por outra AP? \\ QC06. Quem define as atividades pedagógicas em uma AP? \\ QC07. Quem participa de uma mediação pedagógica?}

\subsection{Formalização}

Nesta fase, capturamos a conceituação do domínio com base nas questões de competência. O primeiro passo para a realização desta tarefa, é a produção de um modelo conceitual e SABiO recomenda a linguagem OntoUML para isto. A Figura 1 apresenta o modelo que criamos para representação do domínio de APs e do recorte do domínio de ambientes educacionais, útil ao nosso propósito. Posteriormente se faz necessário a criação de um dicionário de termos e a definição de axiomas para descrever restrições que não podem ser reconhecidas apenas com o desenho do modelo.

Os conceitos apresentados neste modelo foram extraídos da literatura, de entrevistas com especialistas da área e da experiência acadêmica dos engenheiros de ontologia. É importante que seja feito dessa forma, para de fato prover uma conceituação compartilhada sobre este domínio, e não apenas uma conceituação oriunda do nosso conhecimento individual.

Dentre os conceitos apresentados no módulo de ambientes educacionais (desenhado na cor cinza no modelo da Figura 1), destacamos o agente, que pode ser humano ou sintético, como um robô ou software, e o agente humano pode assumir o papel de professor, aluno ou pesquisador.

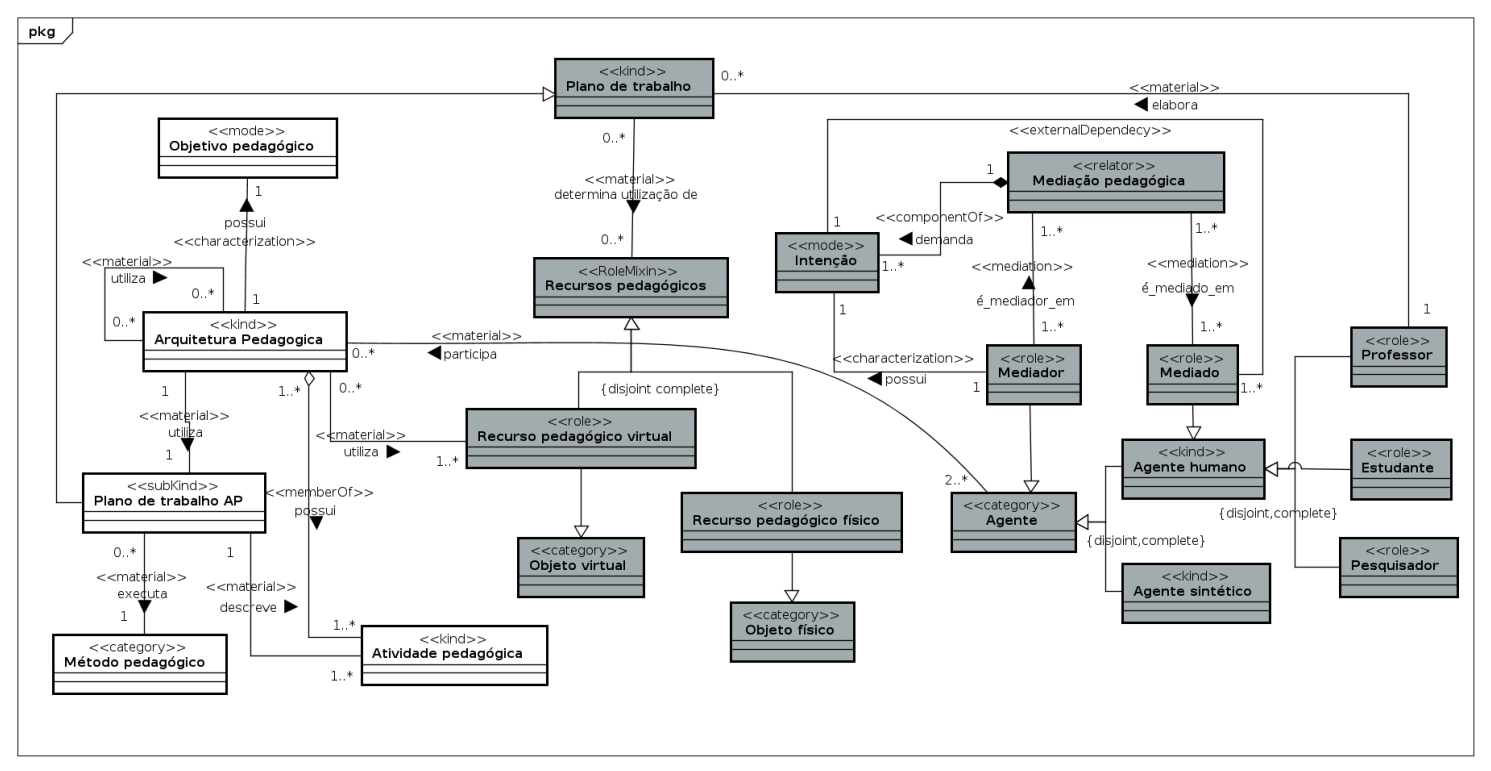

Figura 1. Modelo OntoUML de OAP

Nesta parte do domínio, apenas o professor tem dependência de relacionamento (com Plano de Trabalho). No entanto, os outros conceitos também têm dependências fora dessa representação. Por fim, uma mediação pedagógica deve ter a participação de pelo menos um mediado e um mediador. Um mediado deve ser necessariamente humano e os mediadores podem ser qualquer agente. Assim, o seguinte axioma é válido: 
(A1) Em uma mediação, o mediador e o mediado não podem ser o mesmo agente.

$$
\begin{gathered}
\forall(m c \in \text { Mediação_Pedagogica, } m r \in \text { Mediador, } m d \in \text { Mediado }) \\
\left.\left(\dot{e} \_ \text {Mediador_(mr,mc }\right) \wedge e ́ \_ \text {Mediado_em }(m d, m c) \rightarrow m r \neq m c\right)
\end{gathered}
$$

Cada AP tem um objetivo pedagógico descrito de forma textual sobre o que se pretende alcançar com sua utilização. As APs podem ser implementadas a partir de outras APs e são executadas a partir da descrição de um plano de trabalho que, por sua vez, segue um método pedagógico único até o final de sua execução. Em um AP pode haver a participação de agentes humanos e sintéticos, mas pelo menos dois agentes. O seguinte axioma deve ser considerado:

(A2) Toda AP tem a participação de pelo menos um agente humano.

$\forall$ ap (Arquitetura_Pedagogica(ap) $\rightarrow \exists h(\operatorname{Humano}(h) \wedge \operatorname{participas}(h, p a))$

\subsection{Verificação e Validação}

Para avaliação de OAP, realizamos as atividades de Verificação e Validação (V\&V) da ontologia em duas etapas, considerando as diretrizes propostas pelo método $\mathrm{SABiO}$. As duas etapas são realizadas manualmente na ontologia de referência.

Adaptando o método de avaliação realizado por [Souza et al. 2017], primeiramente realizamos uma atividade de verificação por meio de perícia, na qual averiguamos se os conceitos, relações e axiomas definidos por OAP são capazes de responder questões de competência, em uma abordagem de avaliação por um ser humano [Brank et al. 2005]. Isso é feito por meio de tabelas de verificação de competência. A Tabela 2 mostra as questões de competência e as listas dos elementos da ontologia (conceitos, relacionamentos, propriedades e axiomas) necessários para responder a cada QC de OAP.

\begin{tabular}{|c|c|c|}
\hline QC & Conceitos, $\underline{\text { Relacionamentos e Propriedades }}$ & Axiomas \\
\hline QC01 & Agente participa de Arquitetura pedagógica & A2 \\
\hline \multirow{3}{*}{ QC02 } & Arquitetura pedagógica usa Recurso virtual pedagógico & \\
\hline & Arquitetura pedagógica $\underline{u s a}$ Plano de trabalho AP & \\
\hline & Arquitetura pedagógica possui Atividade pedagógica & \\
\hline QC03 & Arquitetura pedagógica possui Objetivo Pedagógico & - \\
\hline QC04 & Arquitetura pedagógica executa Método Pedagógico & - \\
\hline QC05 & Arquitetura pedagógica $u s a$ Arquitetura pedagógicas & - \\
\hline QC06 & Plano de trabalho descreve Atividade pedagógica & - \\
\hline \multirow{2}{*}{ QC07 } & Mediado é_mediado_em Mediação pedagógica & \multirow{2}{*}{ A1 } \\
\hline & Mediador é_mediador_em Mediação pedagógica & \\
\hline
\end{tabular}

Tabela 2. Verificação de competências de OAP

Uma ontologia de referência deve ser capaz de representar situações do mundo real. Dessa forma instanciamos conceitos e relacionamentos de OAP usando dados de teste extraídos de um projeto real, em uma abordagem baseada em dados para avaliação de ontologia [Brank et al. 2005]. Isso é feito por meio de tabelas de instanciação. Os dados apresentados na Tabela 3 foram extraídos de um projeto concreto para execução de um debate de teses em um curso com alunos de nível de graduação. 
Tabela 3. Instanciação de OAP

\begin{tabular}{|c|c|}
\hline Conceito & Instâncias \\
\hline Arquitetura Pedagógica & Debate de Teses [Nevado et al. 2012]. \\
\hline Objective pedagógico & $\begin{array}{l}\text { Causar desequilíbrio nas certezas provisórias (etapas } 1 \text { e } 2 \\
\text { de PTAP), seguido de adaptação e equilíbrio (etapas } 3 \text { e } 4 \text { ). }\end{array}$ \\
\hline Atividade pedagógica & $\begin{array}{l}\text { Passo } 1 \text { - Escreva a posição inicial sobre uma tese; } \\
\text { R1 - As etapas devem ser abertas em até } 1 \text { dia. }\end{array}$ \\
\hline Método pedagógico & Construtivismo piagetiano. \\
\hline Plano de trabalho PA & $\begin{array}{l}\text { PTAP - Elaborado pelo professor P1: Passo } 1 \text { - Escreva a } \\
\text { posição inicial sobre uma tese; Etapa } 2 \text { - revisão por pares; } \\
\text { Etapa } 3 \text { - Realização de autocrítica a partir da leitura dos } \\
\text { comentários; Etapa } 4 \text { - Escreva a posição final sobre a tese. } \\
\text { Restrições: R1 - As etapas devem ser abertas em até } 1 \text { dia. } \\
\text { R2 - A etapa } 2 \text { deve ser do tipo blind review. }\end{array}$ \\
\hline
\end{tabular}

\section{Especializando OAP}

Para o presente estudo de caso elegemos a AP Debate de Teses [Nevado et al. 2012], por se tratar de uma arquitetura muito difundida na comunidade científica, discutido e publicado em diversos trabalhos [Nevado et al. 2012], [Menezes et al. 2021], [Panceri and Menezes 2014], [Vieira and Tavares 2015]. Esta arquitetura tem como objetivo incentivar que um indivíduo construa conhecimentos sobre um determinado "micromundo" ou construto teórico, apoiado por uma rede de interações. A arquitetura tem como ponto de partida o conhecimento prévio dos participantes sobre o micromundo considerado. As diversas compreensões reveladas pelos participantes geram um debate coletivo que fomenta o confronto de ideias e a reflexão sobre elas, desenvolvido em diferentes etapas, descritas a seguir:

1. Levantamento de teses: os participantes produzem, em conjunto com o professor, afirmações (teses) sobre o micromundo ou conceitos em estudo a partir dos seus conhecimentos prévios, ou seja, sem consulta a fontes externas. As teses devem expressar os conhecimentos atuais, ainda que esses sejam equivocados ou ambíguos. A mediação do professor tem início no momento de definição das teses, quando incentiva que os participantes analisem, de forma conjunta, a potencialidade da tese para produzir um debate;

2. Posicionamento e argumentação individual: os participantes expressam a concordância, discordância ou a dificuldade em decidir com respeito a cada uma das teses. Todos os posicionamentos devem ser apoiados em argumentações;

3. Revisão por pares: após a etapa do posicionamento inicial, os participantes revisam os posicionamentos de um número estabelecido de colegas, tomando-se o cuidado para que não haja a formação de ilhas. Não se trata de concordar ou discordar das argumentações, mas de analisar a sua consistência e problematizá-las. Nesse momento, a mediação distribuída tem um papel essencial, problematizando as ideias em circulação. O professor acompanha os debates e busca incentivar a participação de todo o grupo. A partir da etapa de revisão, são disponibilizadas leituras e discussões que visam trazer novos elementos teóricos para o apoio às reconstruções;

4. Réplica: o argumentador pode concordar ou não com as análises realizadas pelos 
colegas, reafirmando a sua posição inicial ou modificando as suas ideias a partir das contribuições dos revisores;

5. Posicionamento final: nesta etapa os participantes voltam a posicionar-se, reafirmando seu posicionamento (ainda que enriquecido por novos argumentos) ou modificando-o a partir da construção de novos conhecimentos;

6. Avaliação do processo individual e coletivo: na etapa final, os participantes são convidados a apresentar suas reflexões sobre o seu processo (metarreflexões) e sobre o desenvolvimento da arquitetura.

As ontologias de referência, ao contrário das operacionais, devem ser usadas de maneira off-line (sem a necessidade de processamento computacional) para auxiliar os seres humanos em tarefas como negociação de significado, estabelecimento de consenso e facilitação do reuso para outros domínios [Falbo 2014].

OAP é uma ontologia de referência, e pode ser usada como uma meta-ontologia para especializar APs específicas e assim produzir modelos conceituais que possam ser usados, por exemplo, como projeto para construção de software, no caso concepções tecnológicas para favorecer a realização das APs. A Figura 2 mostra um exemplo disso, com um modelo conceitual do Debate de Teses como uma especialização de uma AP.

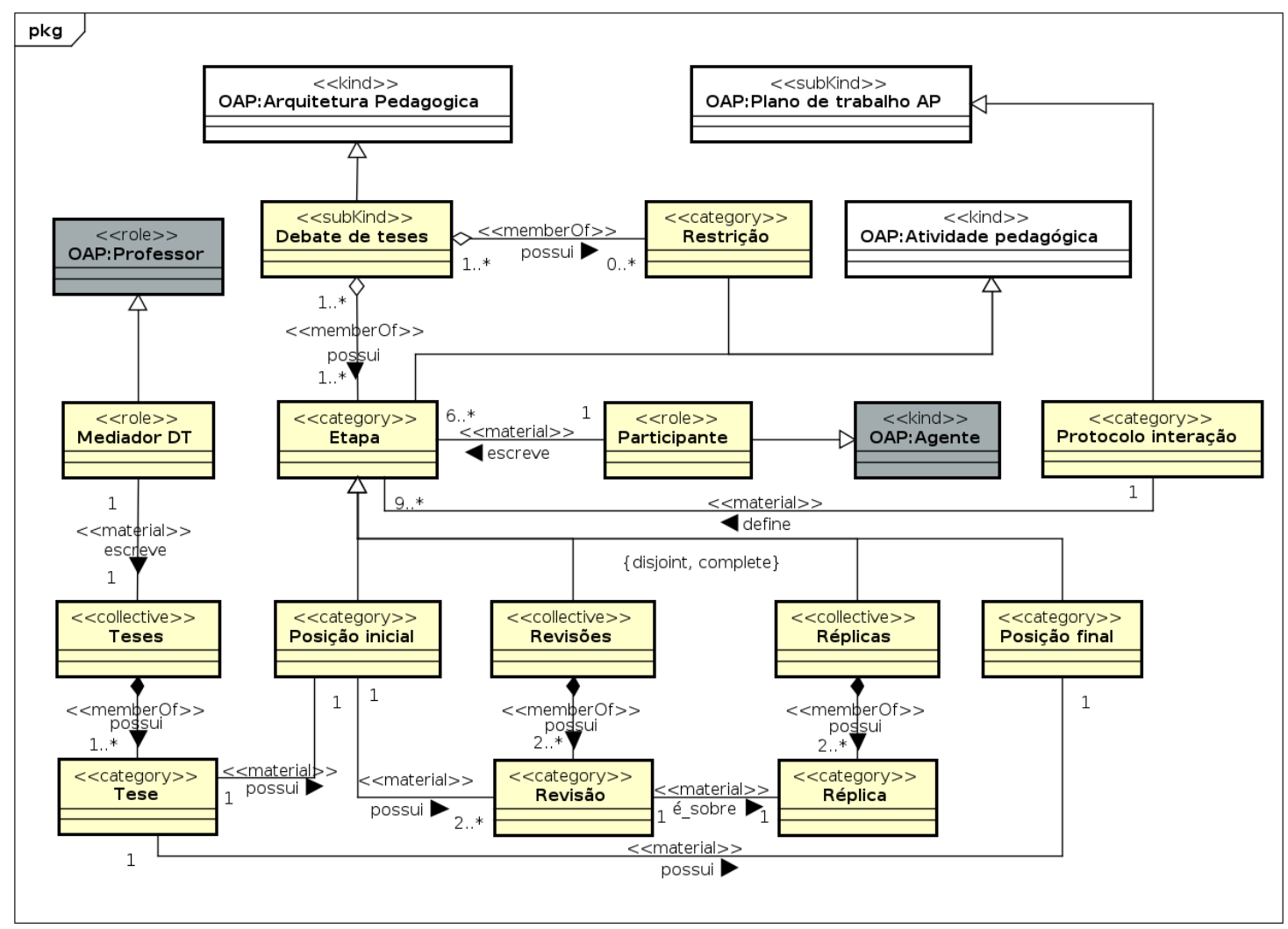

Figura 2. Debate de Teses enquanto uma especialização de OAP

As ontologias podem ser usadas para uma variedade de propósitos, incluindo o suporte à especificação e implementação de qualquer sistema de computação complexo [Duarte and Falbo 2000]. Na Figura 2, observa-se que o modelo de representação do Debates de Teses, pode ser usado tanto como modelo de dados quanto como uma especificação das classes do padrão de orientação a objeto. 
Com a especialização para o Debate de Teses, mostramos como atender o primeiro uso pretendido de OAP, no apoio à especialização de arquiteturas pedagógicas e na geração do modelo de referência para apoiar a construção de software (concepção tecnológica da AP). Ainda, [Menezes et al. 2021] apresentam diversas arquiteturas pedagógicas como por exemplo: (i) Construção cooperativa de programas de computador, (ii) Projetos de aprendizagem, (iii) Portfólio de aprendizagens, (iv) Workshop de avaliação integrada, dentre outras, que podem ser especializadas a partir de OAP, sem maiores dificuldades.

O segundo uso pretendido pode ser realizado ao executarmos uma tarefa inversa à da especialização. Se temos à mão um modelo similar ao da Figura 2, conseguimos identificar elementos que demonstram que o Debate de Teses é de fato uma AP. Por exemplo, é perceptível que as "etapas" de DT constituem "atividades pedagógicas" de AP. Analogamente o "Protocolo de interações" de DT, que no modelo determina como, quando e por quem cada etapa vai ser executada, constitui o "Plano de trabalho" de uma AP.

\section{Considerações finais}

Neste artigo, apresentamos uma ontologia de referência para descrever o domínio de arquiteturas pedagógicas (OAP) utilizando o método $\mathrm{SABiO}$ de engenharia de ontologias. Expomos e descrevemos o modelo conceitual de OAP, realizamos as tarefas de verificação e validação necessárias para avaliar a ontologia e mostramos como usá-la.

Nossa contribuição está no oferecimento de um suporte conceitual para que pesquisadores da área de informática na educação com interesse em arquiteturas pedagógicas, possam especializar novas APs sem a necessidade de um especialista de domínio, e também para que possam comparar o projeto de suas abordagens (que acreditam que sejam APs) com o que de fato seja uma AP e assim balizarem seus trabalhos.

Como trabalhos futuros, pretendemos realizar uma extensão da ontologia para descrever o suporte computacional para a AP especializada a partir de OAP, e assim ajudar na escolha de tecnologias a partir da abordagem utilizada. Ainda temos a intenção de implementar um ambiente de desenvolvimento integrado para facilitar a construção de suportes computacionais para APs, baseado na ontologia.

\section{Referências}

Aragón, R. (2016). Interação e mediação no contexto das arquiteturas pedagógicas para a aprendizagem em rede. Revista de Educação Pública, 25(59/1):261-275.

Bateson, G. (2000). Steps to an ecology of mind: Collected essays in anthropology, psychiatry, evolution, and epistemology. University of Chicago Press.

Brank, J., Grobelnik, M., and Mladenic, D. (2005). A survey of ontology evaluation techniques. In Proceedings of the conference on data mining and data warehouses (SiKDD 2005), pages 166-170. Citeseer Ljubljana, Slovenia.

Carvalho, M. J. S., de Nevado, R. A., and Menezes, C. S. (2005). Arquiteturas pedagógicas para educação à distância: concepções e suporte telemático. In Brazilian Symposium on Computers in Education (Simpósio Brasileiro de Informática na Educação-SBIE), volume 1, pages 351-360. 
Castro, A. and Menezes, C. (2011). Aprendizagem colaborativa com suporte computacional. Pimentel, M. e Fuks, H. Sistemas Colaborativos. Rio de Janeiro: Campus. ISBN, pages $978-85$.

Duarte, K. C. and Falbo, R. A. (2000). Uma ontologia de qualidade de software. In Workshop de Qualidade de Software, João Pessoa, pages 275-285.

Falbo, R. d. A. (2014). Sabio: Systematic approach for building ontologies. In ONTO. COM/ODISE@FOIS.

Freire, P. (2014). Por uma pedagogia da pergunta. Editora Paz e Terra.

Guizzardi, G. (2007). On ontology, ontologies, conceptualizations, modeling languages, and (meta) models. Frontiers in artificial intelligence and applications, 155:18.

Jackson, N. J. (2013). Learning ecology narratives. Lifewide Learning, Educafion and Personal Development e-book (1-26).

Lévy, P. (1993). As tecnologias da inteligência: O futuro do pensamento na era da informática. Editora 34.

Marcon, K., Machado, J. B., and Carvalho, M. J. S. (2013). Arquiteturas pedagógicas e redes sociais: uma experiência no facebook. Revista de Informática Aplicada, 9(2).

Menezes, C. S. d., Castro-Junior, A. N. d., and Aragon, R. (2021). Arquiteturas Pedagógicas para Aprendizagem em Rede. Série de livrostexto da CEIE-SBC. Disponível em https://ieducacao.ceie-br.org/ arquiteturas-pedagogicas/. Acessado em 21-08-2020.

Nevado, R. A., Menezes, C. S., and Júnior, R. R. V. (2012). Debate de teses-uma arquitetura pedagógica. In Brazilian Symposium on Computers in Education (Simpósio Brasileiro de Informática na Educação-SBIE), volume 1.

ONTOUML (2018). OntoUML specification document, and pattern catalogue. Disponível em: https://ontouml.readthedocs.io/en/latest/index. html. Acessado em 16-06-2020.

Panceri, S. S. and Menezes, C. S. (2014). Alpes: Um sistema multiagente para análise de produções textuais no contexto de um debate de teses. In Brazilian Symposium on Computers in Education (Simpósio Brasileiro de Informática na Educação-SBIE), volume 25 , page 45 .

Piaget, J. (2016). L'epistemologia genetica. Edizioni Studium Srl.

Souza, É. F. d., Falbo, R. d. A., and Vijaykumar, N. L. (2017). Roost: reference ontology on software testing. Applied Ontology, 12(1):59-90.

Valente, J. A., de Almeida, M. E. B., and Geraldini, A. F. S. (2017). Metodologias ativas: das concepções às práticas em distintos níveis de ensino. Revista Diálogo Educacional, 17(52):455-478.

Vieira, R. R. M. and Tavares, O. d. L. (2015). Modelo para descrição de espaços colaborativos: Apoiando a realização de arquiteturas pedagógicas. Sistemas Colaborativos, pages 189-196. 